\title{
Analysis the Performance of Transceiver Circuits for Pulse Based Ultra Wideband
}

\author{
Bobby Barua, Member IACSIT and IEEE
}

\begin{abstract}
Ultra-wideband is a technology that can be use at very low energy levels for short-range high bandwidth communications by using a large portion of the radio spectrum. In this paper we is specifically focused on the performance of software radio transceiver design for impulse-based UWB with the ability to transmit a raw data rate of 100 Mbps yet encompasses the adaptability of a reconfigurable digital receiver. To complete the analysis we introduce a transmitter and receiver of pulse based ultra wideband modulation. Direct sequence spread spectrum has become the modulation method of choice for wireless local area networks, and personal communication systems, because it's numerous advantages such as jammer suppression, code division multiple access, and ease of implementation. Again spread spectrum techniques are most favourable for indoor communication needs and digital radio links, where most of the applications are found. We also observe its characteristics and complete the modulation techniques. Finally we examine UWB communication systems paying particular attention to transmitter and receiver design.
\end{abstract}

Index Terms - Code division multiple access (CDMA). direct sequence spread spectrum (DSSS), personal communication systems (PCS), ultra-wideband (UWB), wireless local area networks (WLAN's).

\section{INTRODUCTION}

Ultra wideband (also known as UWB or as digital pulse wireless) is a wireless technology for transmitting large amounts of digital data over a wide spectrum of frequency bands with very low power for a short distance. Ultra wideband radio not only can carry a huge amount of data over a distance up to 230 feet at very low power (less than 0.5 milliwatts), but has the ability to carry signals through doors and other obstacles that tend to reflect signals at more limited bandwidths and a higher power. The concept of UWB was formulated in the early $1960 \mathrm{~s}$ through research in time-domain electromagnetic and receiver design, both performed primarily by Gerald F. Ross[1]. Through his work, the first UWB communications patent was awarded for the short-pulse receiver, which he developed while working for Sperry Rand Corporation. Throughout that time, UWB was referred in broad terms as "carrier less" or impulse technology. After that UWB was coined in the late 1980s to describe the development, transmission, and reception of ultra-short pulses of radio frequency (RF) energy. For communication applications, high data rates are possible due to the large number of pulses that can be created in short time duration [2]. A number of various receiver design

Manuscipt received September 13, 2011; revised December 8, 2011.

Bobby Barua is with the Department of EEE, Ahsanullah University of Science and Technology, Dhaka, Bangladesh. (email: bobby@aust.edu) architectures have been examined to investigate UWB communication performance, most of them hardware based. For data demodulation purposes, hardware implementations such as a sampling bridge circuit and a matched filter are frequently used. The primary disadvantage of the sampling bridge circuit is the loss of data rate, as it undersamples the incoming pulse with a sliding sampling position, taking a series of snapshots to produce a final waveform. Therefore, multiple pulses per bit are required to transmit data, limiting the maximum data rate [3]. To perform data demodulation using a matched filter in hardware, an estimated received pulse must be generated at the receiver and correlated with the incoming waveform. A system with this architecture must have a highly stable oscillator and precise timing accuracy [4]. Other receiver performance improvements, such as a Rake receiver, which is necessary to capture multipath to create adequate signal energy, are difficult to implement using hardware [5],[6]. Multiple correlators are necessary to capture each multipath with a channel estimator running in parallel to detect the time when each correlator should be triggered and to determine channel polarity. Timing accuracy is again an issue when the pulse rate is high, as data processing time and signal propagation delay become problems. Additional drawbacks include extra hardware and the limited number of Rake fingers due to the fixed number of correlators [7-8]. Again a significant difference between traditional radio transmissions and UWB radio transmissions is that traditional systems transmit information by varying the power level, frequency, and/or phase of a sinusoidal wave [9]. UWB transmissions transmit information by generating radio energy at specific time instants and occupying large bandwidth thus enabling a pulse-position or time-modulation.UWB communications transmit in a way that doesn't interfere largely with other more traditional 'narrow band' and continuous carrier wave uses in the same frequency band [10]. However first studies show that the rise of noise level by a number of UWB transmitters puts a burden on existing communications services [11],[12]. This may be hard to bear for traditional systems designs and may affect the stability of such existing systems.

In this paper we focused on the performance of a software based radio transceiver for impulse-based UWB with the ability to transmit a raw data rate of $100 \mathrm{Mbps}$ yet encompass the adaptability of a reconfigurable digital receiver. We also discussed various modulation schemes that are possible with pulse-based UWB. Additionally, we examined multiple access techniques and receiver architectures suitable for UWB systems. Ultimately, the transceiver design demonstrates the advantages and challenges of UWB technology while boasting high data rate communication capability and providing the flexibility of a research test bed. 


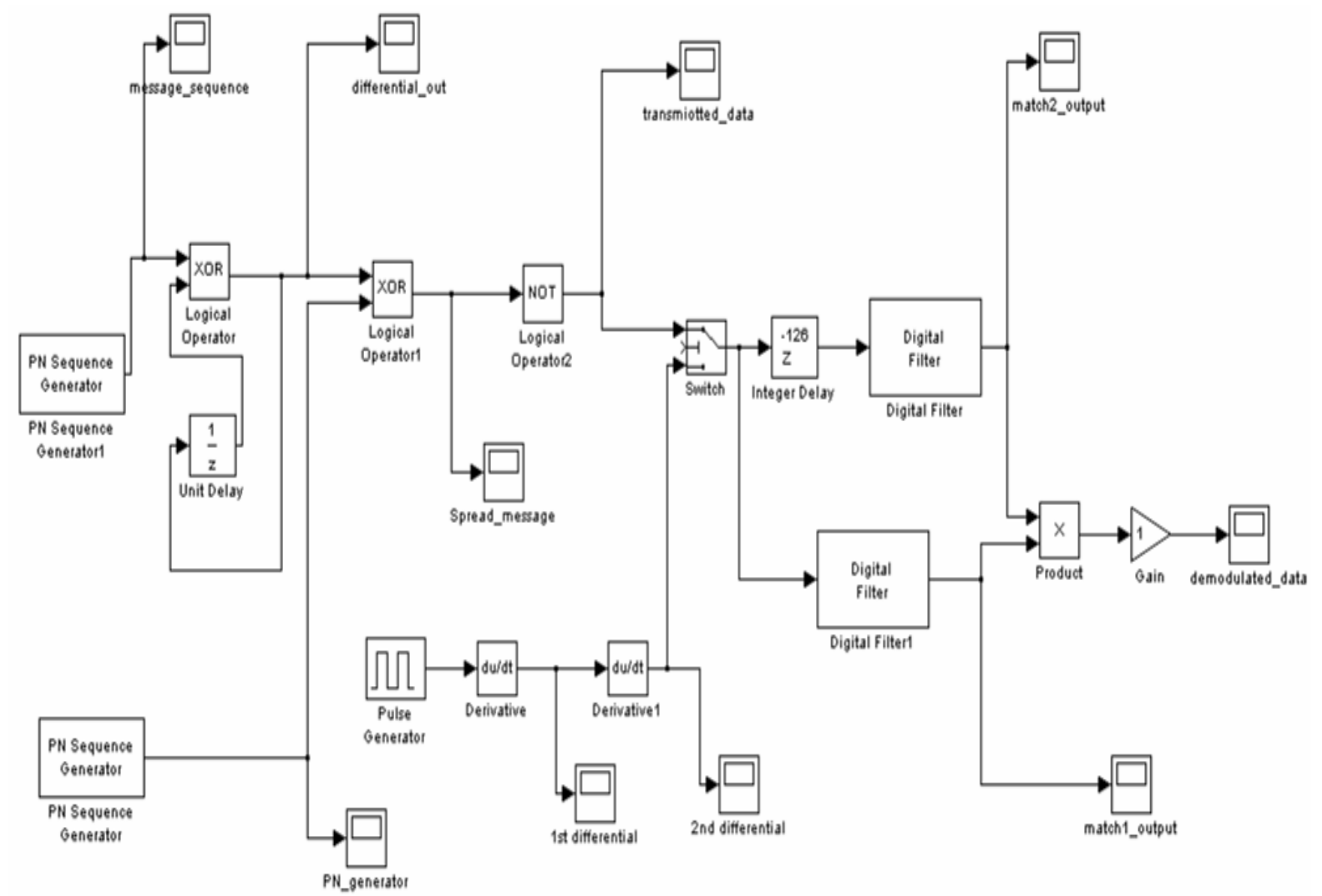

Fig. 1. Simulink model of DPSK DSSS Transceiver.

\section{SYSTEM MODEL}

The designed model for the transceiver is shown in Fig-1, consists of a hierarchical system where blocks represent subsystems and oscilloscopes are placed along the path for display purposes. The main components or blocks of this design are PN sequence generator, XOR, Unite delay, Switch, Pulse generator, Derivative, Integer delay, Digital Filter, Product, Gain and oscilloscope. The PN Sequence Generator block generates a sequence of pseudorandom binary numbers A pseudo noise sequence can be used in a pseudorandom scrambler and descrambler. It can also be used in a direct-sequence spread-spectrum system. The PN Sequence Generator block uses a shift register to generate sequences. Here, PN sequence generator uses for generating both incoming message and high speed pseudo random sequence number for spreading purpose. XOR block work as a mixer, it mixes two different inputs with each other as digital XOR does and gives the output. The Unit Delay block holds and delays its input by the sample period you specify. This block is equivalent to the $\mathrm{z}-1$ discrete-time operator. The block accepts one input and generates one output. Each signal can be scalar or vector. If the input is a vector, the block holds and delays all elements of the vector by the same sample period. Pulse generator capable of generating a variety of pulses with an assortment of options. Switch uses for switching the two different input and direct it to the output as per requirement. Derivative block basically differentiate the input data. The pulse generator and sequentially two derivatives are used for performing Bi-phase modulation as per requirement. Integer delay use for delay the incoming data for 63 chip. Digital filter has its special use. It uses for creating digital filter for recovering purpose. Gain blocks use for amplifying process. Oscilloscopes are placed along the path for display purpose.

Direct-sequence spread spectrum (DSSS) is a modulation technique. The DPSK DSSS modulation and dispread techniques are mainly use for designing the whole transceiver with the exception of receiving the signal using Bi-phase modulation. The design for pulse based UWB is divided into three parts as DSSS DPSK transmitter where transmitter part is separately designed, DPSK DSSS transceiver where received signal has dispread with some propagation delay, DPSK DSSS transceiver with Bi-phase modulator and matched filter where original signal has recovered. The data signal, rather than being transmitted on a narrow band as is done in microwave communications, is spread onto a much larger range of frequencies (RF bandwidth) using a specific encoding scheme. This encoding scheme is known as a Pseudo-noise sequence, or PN sequence. Direct sequence spread spectrum has become the modulation method of choice for wireless local area networks, and personal communication systems. Direct-sequence spread-spectrum transmissions multiply the data being transmitted by a "noise" signal. This noise signal is a pseudorandom sequence of 1 and -1 values, at a frequency much higher than that of 
the original signal, thereby spreading the energy of the original signal into a much wider band. The resulting signal resembles white noise, like an audio recording of "static". However, this noise-like signal can be used to exactly reconstruct the original data at the receiving end, by multiplying it by the same pseudorandom sequence (because $1 \times 1=1$, and $-1 \times-1=1)$. This process, known as "de-spreading", mathematically constitutes a correlation of the transmitted PN sequence with the PN sequence that the receiver believes the transmitter is using. For de-spreading to work correctly, transmit and receive sequences must be synchronized. This requires the receiver to synchronize its sequence with the transmitter's sequence via some sort of timing search process. However, this apparent drawback can be a significant benefit: if the sequences of multiple transmitters are synchronized with each other, the relative synchronizations the receiver must make between them can be used to determine relative timing, which, in turn, can be used to calculate the receiver's position if the transmitters' positions are known. This is the basis for many satellite navigation systems.

The resulting effect of enhancing signal to noise ratio on the channel is called process gain. This effect can be made larger by employing a longer PN sequence and more chips per bit, but physical devices used to generate the PN sequence impose practical limits on attainable processing gain.

\section{DPSK TRANSMITTER}

DPSK DSSS transmitter consists of PN Sequence generator which generates a sequence of pseudo random binary numbers using a linear-feedback shift register, XOR used for mixing data, Unite delay used for delayed data and oscilloscopes are placed along the path for display purposes. Here, PN Sequence generator is used as both generating message and a sequence of pseudo random binary numbers for spreading process. When differentially encoding an incoming message, each input data bit must be delayed until the next one arrives. The delayed data bit is then mixed with the next incoming data bit. The output of the mixer gives the difference of the incoming data bit and the delayed data bit. The differentially encoded data is then spread by a high-speed pseudo noise sequence (PN).This spreading process assigns each data bit. It's own unique code, allowing only a receiver with the same spreading to dispread the encoded data. The 63-bit pseudo noise sequences (PN) used in this papers are generated by a 6th order maximal length sequence shown in equation one.

$$
g(x)=x^{6}+x^{5}+1
$$

Basically, The PN Sequence Generator block generates a sequence of pseudorandom binary numbers. A pseudo noise sequence can be used in a pseudorandom scrambler and descrambler. It can also be used in a direct-sequence spread-spectrum system. The PN Sequence Generator block uses a shift register to generate sequences. If all $r$ registers in the generator update their values at each time step according to the value of the incoming arrow to the shift register and the adders perform addition then the Generator Polynomial parameter, which is a primitive binary polynomial in $z$ will be:

$$
g_{r} z^{r}+g_{r-1} z^{r-1}+g_{r-2} z^{r-2}+\ldots \ldots . .+g_{0}
$$

The coefficient $g_{k}$ is 1 if there is a connection from the $k^{\text {th }}$ register, as levelled in the preceding diagram, to the adder. The leading term $\mathrm{g}_{\mathrm{r}}$ and the constant term $\mathrm{g}_{0}$ of the Generator Polynomial parameter must be 1 .

We can specify the Generator polynomial parameter using either of these formats:

- A vector that lists the coefficients of the polynomial in descending order of powers. The first and last entries must be 1 . Note that the length of this vector is one more than the degree of the generator polynomial.

- A vector containing the exponents of $\mathrm{z}$ for the nonzero terms of the polynomial in descending order of powers. The last entry must be 0 .

The Initial states parameter is a vector specifying the initial values of the registers. The Initial states parameter must satisfy these criteria:

- All elements of the Initial states vector must be binary numbers.

- The length of the Initial states vector must equal the degree of the generator polynomial.

The following table indicates two sets of parameter values that correspond to a generator polynomial of the 63-bit pseudo noise sequences (PN) used in this paper for generating 6 th order maximal length sequence,

TABLE I: TwO SETS OF PARAMETER VALUES THAT CORRESPOND TO A

\begin{tabular}{|c|c|c|}
\hline Quantity & Example1 & Example2 \\
\hline Generator polynomial & $\mathrm{G}_{1}=\left[\begin{array}{lllllll}1 & 1 & 0 & 0 & 0 & 0 & 1\end{array}\right]$ & $\mathrm{g}_{1}=\left[\begin{array}{lll}6 & 5 & 0\end{array}\right]$ \\
\hline $\begin{array}{l}\text { Degree of generator } \\
\text { polynomial }\end{array}$ & 6 , which is $g_{1}-1$ & 6 \\
\hline Initial states & {$\left[\begin{array}{llllll}1 & 1 & 0 & 0 & 0 & 0\end{array}\right]$} & {$\left[\begin{array}{llllll}1 & 1 & 0 & 0 & 0 & 0\end{array}\right]$} \\
\hline
\end{tabular}
Generator Polynomial of The 63-Bit PSEUdo NoISE SEQUENCES

The Shift parameter shifts the starting point of the output sequence. With the default setting for this parameter, the only connection is along the arrow levelled $\boldsymbol{m}_{0}$, which corresponds to a shift of 0 . The parameter is described in greater detail below.

It can be possible to shift the starting point of the PN sequence with the Shift parameter. Specification of parameter in either of two ways:

- An integer representing the length of the shift

- A binary vector, called the mask vector, whose length is equal to the degree of the generator polynomial

The difference between the block's output when it sets 
Shift (or mask) to 0.

The maximal length spreading sequence uses a much wider bandwidth than the encoded data bit stream, which causes the spread sequence to have a much lower power spectral density. The transmitted signal is then given by,

$$
x(t)=m(t) c(t)
$$

where $\boldsymbol{m}(\boldsymbol{t})$ is the differentially encoded data, and $\boldsymbol{c}(\boldsymbol{t})$ is the 63 chip PN spreading code. For recovering of message sequence, we XOR the modulated signal with same type of 63-bit pseudo noise sequences (PN). Here we also use a unite delay to find the original signal. The signal recovering process is successfully done with some propagation delay which was obvious because of some noise \& losses.

\section{DPSK RECEIVER}

Before dispreading, the receiving signal is modulated by Bi-phase modulation technique then signal is split into two parallel paths and fed into two identical matched filters with the input to one having a delay of 63 chips. Certain advantage of Bi-phase modulation is, improvement over OOK and PPM in BER performance, as $E_{b} / N_{0}$ are $3 \mathrm{~dB}$ less than OOK for the same probability of bit error. The probability of bit error for Bi-phase modulation assuming matched filter reception is:

$$
P_{e}=Q\left(\sqrt{\frac{2 E_{b}}{N_{0}}}\right)
$$

Another benefit of Bi-phase modulation is its ability to eliminate spectral lines due to the change in pulse polarity. This aspect minimizes the amount of interference with conventional radio systems. A decrease in the overall transmitted power could also be attained, making Bi-phase modulation a popular technique in UWB systems when energy efficiency is a priority.

Special type of Digital Matched Filter have used for recovering the transmitted message. This Digital matched filtering is a data processing routine which is optimal in term of signal-to-noise ratio (SNR). Specifically, it can be shown for an additive white Gaussian noise (AWGN) channel with no interference that the matched filter maximizes the SNR for a pulse modulated system. To perform this operation, the received waveform is over sampled to allow for multiple samples per pulse period. Over sampling gives a more accurate representation of the pulse shape, which then produces better results using a digital matched filter. Correlation processing, another form of matched filtering, is often used in the digital domain when dealing with white noise channels. The method for calculating the correlation output is the following:

$$
g(k)=\sum_{t=1}^{N-1} r(t) h(t)
$$

where:

$g(k)$ is the resulting correlation value

$\mathrm{k} \quad$ is the $\mathrm{k}^{\text {th }}$ pulse period
$\mathrm{N}$ is the number of samples in one pulse width

$r(t)$ is the received sampled waveform

$h(t)$ is the known pulse waveform

One of the primary drawbacks of the matched filter receiver topology is the lack of knowledge of the pulse shape at the receiver due to distortion in the channel. Imperfect correlations can occur by processing the data with an incorrect pulse shape, causing degradation in correlation energy. There are numerous ways to correct this problem, including an adaptive digital equalizer or matching a template by storing multiple pulse shapes at the receiver. A more accurate approach is to estimate the pulse shape from the pilot pulses, which will experience the same channel distortion as the data pulses. This estimation technique is a promising solution to UWB pulse distortion.

The outputs of the two matched filters are denoted by $x_{1}(t)$ and $x_{2}(t)$ are given by

$$
\begin{gathered}
x_{1}(t)=d\left(t-t_{0}\right) R_{c}(t) \\
x_{2}(t)=d\left(t-t_{0}-T_{b}\right) R_{c}\left(t-T_{b}\right)
\end{gathered}
$$

where $\boldsymbol{T}_{\boldsymbol{b}}$ the data is bit period, and $\boldsymbol{R}_{\boldsymbol{c}}(\boldsymbol{t})$ is the autocorrelation function of the 63-chip pseudorandom sequence. Since there are exactly 63 chips per data bit the PN sequence is periodic with $\boldsymbol{T}_{\boldsymbol{b}}$ so

$$
R(t)=R_{c}\left(t-T_{b}\right)
$$

The two outputs of the matched filters are then mixed and then low pass filtered and the original message is recovered.

The process of deriving the matched filter does not help much in developing an understanding of what the matched filter is all about. There is $\mathrm{n}$ alternative way to look at the matched filter process that is much more intuitive. This method is called Correlation receiver. At first for matched filter we know,

$$
h(t)=k s *\left(t_{d}-t\right)
$$

where, $\boldsymbol{h}(\boldsymbol{t})$ is the impulse response, $\mathrm{k}$ is an arbitrary constant and can be used to represent gain through the signal and $\boldsymbol{t}_{\boldsymbol{d}}$ is single bit period. The output response of matched filter is given by the convolution equation.

$$
y(t)=\int_{-\infty}^{\infty} x(\lambda) h(t-\lambda) d \lambda
$$

For a single bit period $\boldsymbol{t}_{d}$,

$$
y(t)=\int_{0}^{t_{d}} x(\lambda) h(t-\lambda) d \lambda
$$

Now lets take the impulse response $\boldsymbol{h}(\boldsymbol{t})$ and shift it by $\lambda$ so we can plug in its shifted value in the above equation. Correspondingly the right sight shift by $+\lambda$. The important thing is that the time shift in $\boldsymbol{h}(\boldsymbol{t})$ has to be opposite to the time shift in $s(t)$ for a matched filter 


$$
h(t-\lambda)=k s *\left(t_{d}-t+\lambda\right)
$$

As we know the output of matched filter is

$$
s_{0}\left(t_{d}\right)=\frac{1}{2 \pi} \int_{-\infty}^{\infty}|s(\omega)| d \omega
$$

And the mean square noise output of the matched filter is

$$
\overline{n_{0}^{2}}(t)=\frac{N_{0}}{2}
$$$$
\text { Which give us, } \quad S N R=\frac{2 E}{N_{0}}
$$

Now substituting SNR into equation 14 into eqation 13, we have

$$
y(t)=\frac{2 k}{N_{0}} \int_{0}^{t_{d}}[s(\lambda)+n(\lambda)] s\left(t_{d}-t+\lambda\right) d \lambda
$$

Now set $\boldsymbol{t}=\boldsymbol{t}_{\boldsymbol{d}}$ and we can do that because it we are only interested in the result of this operation at the precise moment when $\boldsymbol{t}=\boldsymbol{t}_{\boldsymbol{d}}$ or the end of the bit time when we will make a decision about what was sent.

$$
y(t)=\frac{2 k}{N_{0}} \int_{0}^{t_{d}}[s(\lambda)+n(\lambda)] s(\lambda) d \lambda
$$

The above expression is exactly the same as doing cross-correlation between the input signal which includes noise and the original signal without noise. The leads us to an alternative way to implement a matched filter, called the correlator. The correlation receiver is exactly the same thing mathematically as the matched filter but much easier to comprehend and much easier to implement in hardware.

\section{RESULTS AND DISCUSSION}

Following the analytical approach presented in section III and IV, we evaluate the simulation result of UWB technology. The simulations are performed using matlab, and the proof-of-concept is slightly worse than theoretical values for a perfectly matched receiver due to the imperfections in the template caused by noise and aperture delay variation. Fig. 2 shows the original input message sequence that is generated from a PN sequence generator.

Then, the incoming messages are differentially encoded by using mixer and unit delay. Eventually the mixer will give the difference of the incoming data bit and the delayed data bit. The differentially encoded data is then spread by a high-speed 63-bit pseudo noise (PN) Sequence generator which is generated by a 6 th order maximal length sequence. Here, 63 bit pseudo noise sequences (PN) have been used for generating both the message and high frequency carrier signal. Instead of using 63 bit PN sequence different PN sequence generator could be used for more successful data transmission and data recovery purpose.

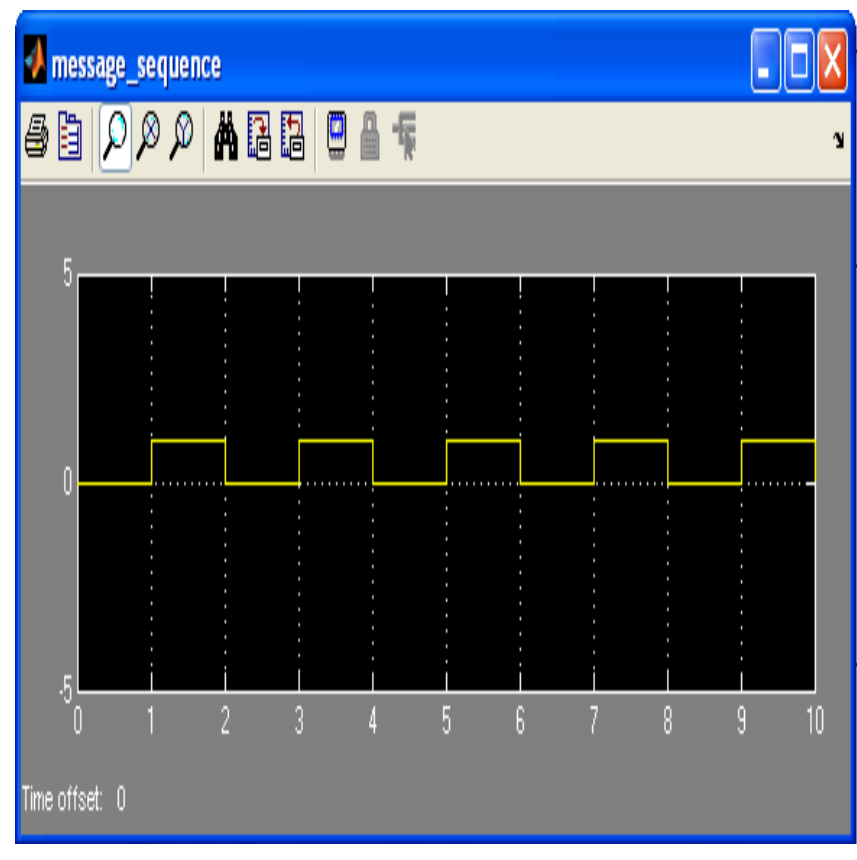

Fig. 2. Original transmitted message signal.

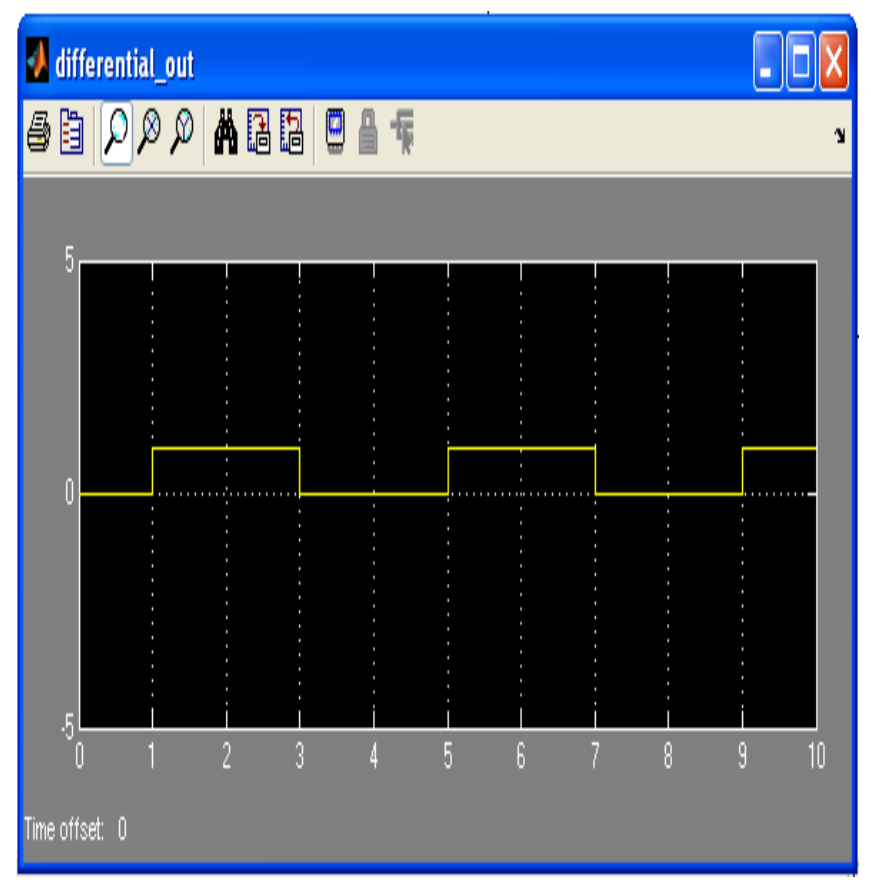

Fig. 3. Differential output of original message signal

Fig. 3 shows such a differential output of the original message signal. Fig. 4 displayed the $1^{\text {st }}$ differential data and Fig. 5 displayed the $2^{\text {nd }}$ differential data.

One important thing is that two 63-chip FIR filters were used as matched filters in both arms of the DPSK receiver. There are various useful reasons for the use of matched filter. Among these the most important are matched filter is kind of an averaging filter which maximizes the signal to noise ratio of received signal, it also minimizes the BER of the signal and two symmetrical filters make a matched pair in which one to transmit and other to receive. 


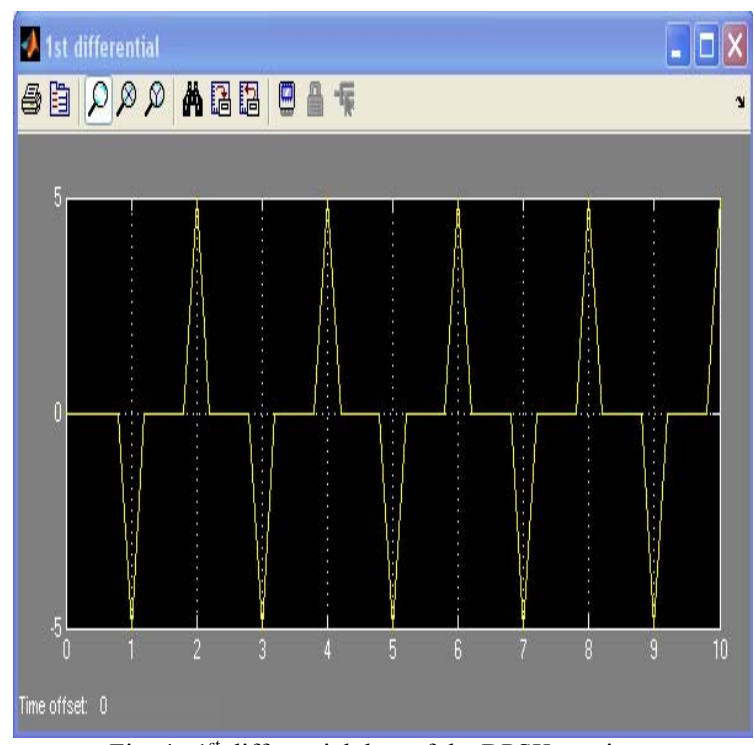

Fig. 4. $1^{\text {st }}$ differential data of the DPSK receiver.

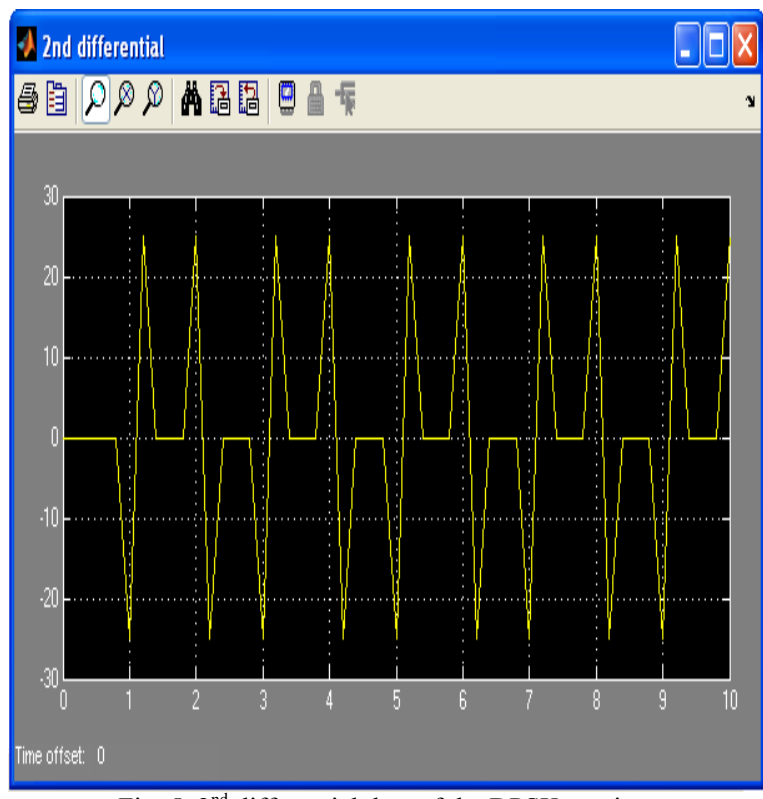

Fig. $5.2^{\text {nd }}$ differential data of the DPSK receiver

Fig.6 displayed the $1^{\text {st }}$ match filter output and Fig. 7 displayed the $2^{\text {nd }}$ match filter output.

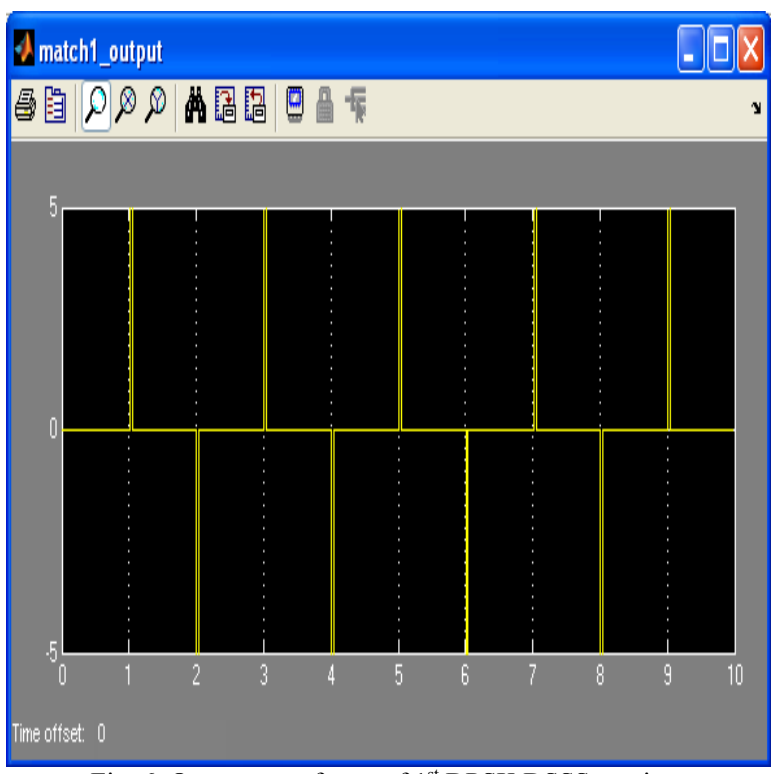

Fig. 6. Output waveforms of $1^{\text {st }}$ DPSK DSSS receiver

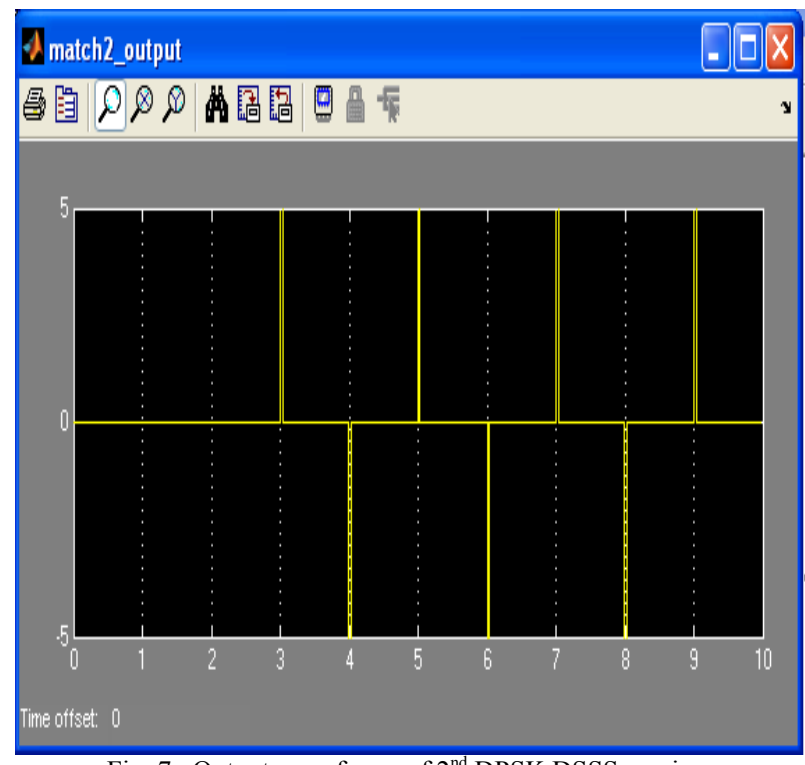

Fig. 7. Output waveforms of $2^{\text {nd }}$ DPSK DSSS receiver

This spreading process assigns each data bit its own unique code which is shown in Fig. 8., allowing only a receiver with the same spreading to dispread the encoded data.

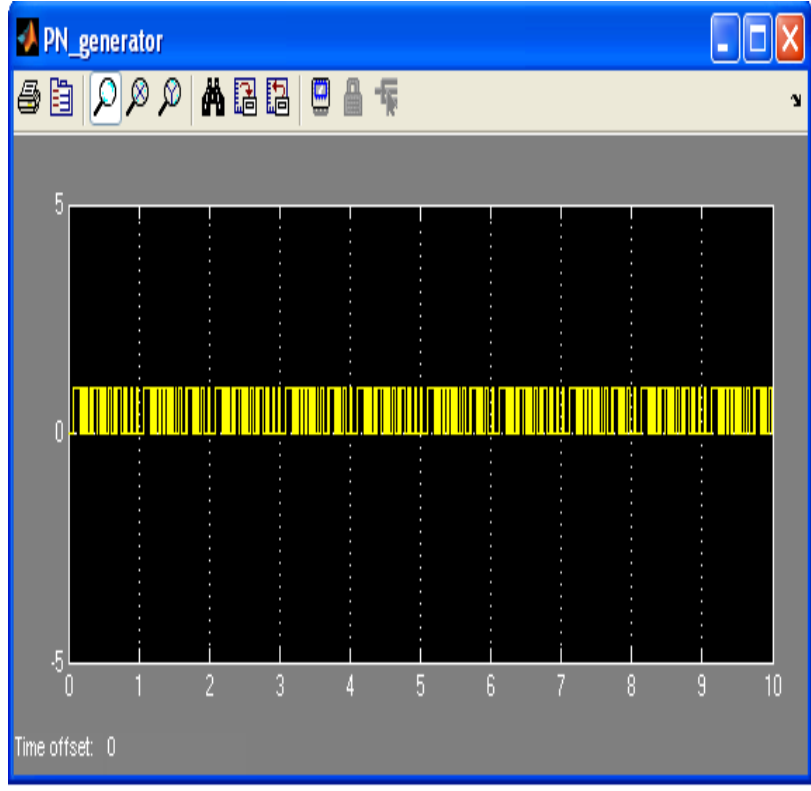

Fig. 8. Output waveforms of Simulink DPSK DSSS transmitter

For recovering of message sequence in the receiving part of DPSK DSSS transceiver, the modulated signal has been dispread using same type of 63-bit pseudo noise sequences and also use a unite delay to find the original signal. Before dispreading, the receiving signal is modulated by Bi-phase modulation technique then signal is split into two parallel paths and fed into two identical matched filters with the input to one having a delay of 63 chips.

Among two split signal, one is spreading received message and another is Bi-phase modulated signal. The signal recovering process is successfully done with some propagation delay which was obvious because of some noise $\&$ losses. Fig. 9 denoted the differential output of receiver side and Fig. 10 shows the recovered messages. 


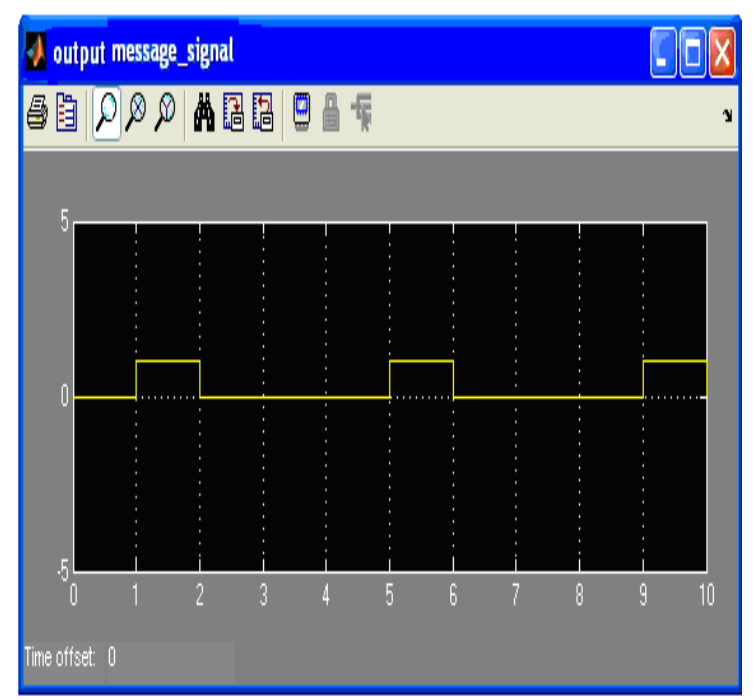

Fig. 9. Actual Output message signal of Simulink DPSK DSSS receiver

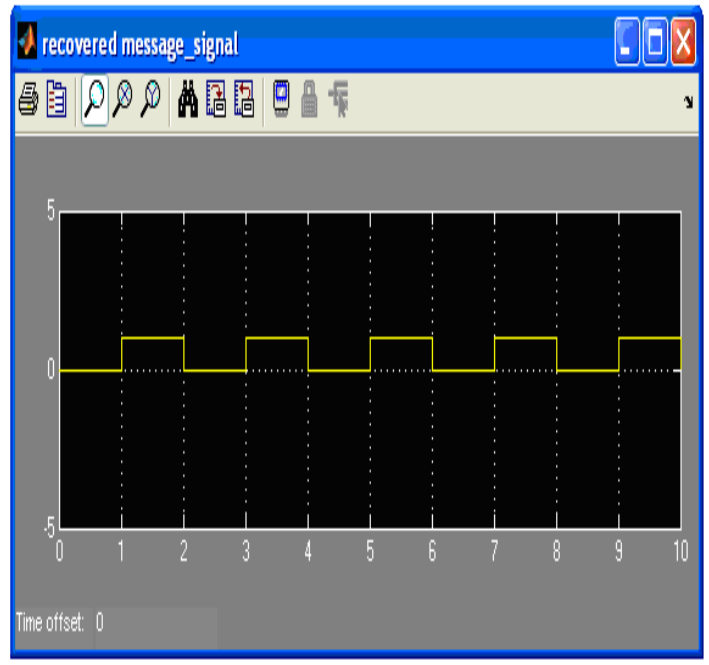

Fig.10. Recovered output message signal

\section{CONCLUSIONS}

The performance of UWB technology is evaluated using Time Hopping (TH) technique. The main key facts of impulse based ultra wide band transceiver are PS sequence generator and the matched filter. Here, 63 bit pseudo noise sequences (PN) have been used for generating both the message and high frequency carrier signal. The matched filter is used as correlator in which cross-correlation is occurred between the input signals. Another noticeable point is use of more matched filter could increase the efficiency of the transceiver. In this paper we only include two matched filters for recovery. More use of matched filter open the source of matching the signal more accurately. It is also found that by increasing the number of averaged pilot pulses in the pilot-based matched filter template, better performance can be obtained, although the data rate will suffer. Finally, use of the template sliding matched filter synchronization routine led to worse BER performance when compared with perfect synchronization results. The results of the system simulation give a solid foundation for the design as a whole, but also will assist in the future with issues such as the implementation of receiver algorithms within the FPGA and determining timing limitations when the receiver is being constructed.

\section{REFERENCES}

[1] G. F. Ross, "Transmission and reception system for generating and receiving base-band duration pulse signals without distortion for short base-band pulse communication system," US Patent 3,728,632, April 17, 1973.

[2] Authorization of Ultra wideband Technology, First Report and Order, Federal Communications Commission, February 14, 2002.

[3] C. R. Anderson, "Ultra wideband Communication System Design Issues and Tradeoffs," Ph.D. Qualifier Exam, Virginia Polytechnic Institute and State University, May 12, 2003.

[4] C. R. Anderson, A. M. Orndorff, R. M. Buehrer, and J. H. Reed, “An Introduction and Overview of an Impulse-Radio Ultra wideband Communication System Design," tech. rep., MPRG, Virginia Polytechnic Institute and State University, June 2004.

[5] S. Licul, J. A. N. Noronha, W. A. Davis, D. G. Sweeney, C. R. Anderson, T. M. Bielawa, "A parametric study of time-domain characteristics of possible UWB antenna architectures," submitted to IEEE Vehicular Technology Conference, February 2003.

[6] R. Blazquez et al., "Digital Architecture for an Ultra-wideband Radio Receiver," VTC., Oct. 2003, pp. 1303-07.

[7] J. S. Lee, C. Nguyen, and T. Scullion, "New uniplanar subnano second monocycle pulse generator and transformer for time-domain microwave applications," IEEE Transactions on Microwave Theory and Techniques, Vol. 49, No. 6, pp. 1126- 1129, June 2001.

[8] J. Han and C. Nguyen, "A new ultra-wideband, ultra-short monocycle pulse generator with reduced ringing," IEEE Microwave and Wireless Components Letters, Vol. 12, No. 6, pp. 206-208, June 2002.

[9] J. R. Foerster, "The performance of a direct-sequence spread ultra-wideband system in the presence of multipath, narrowband interference, and multiuser interference," IEEE Conference on Ultra Wideband Systems and Technologies, May 2002.

[10] F. S. Lee, D. D. Wentzloff, and A. P. Chandrakasan, "An Ultra-Wideband Baseband Front-End," Radio Freq. IC Symp., June 2004, pp. 493-96.

[11] D. D. Wentzloff and A. P. Chandrakasan, "A 3.1-10.6 GHz Ultra-Wideband Pulse-Shaping Mixer,” RFIC Symp., June 2005.

[12] R. Blazquez and A. Chandrakasan, "Architectures for Energy-Aware Impulse UWB Communications," IEEE Int'l. Conf. Acoustics, Speech, and Sig. Proc., Mar. 2005, pp. 789-92.

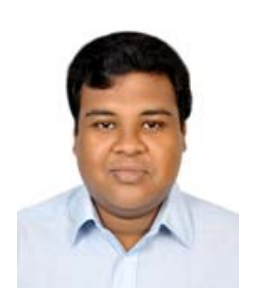

Bobby Barua is Assistant Professor, Department of Electrical and Electronic Engineering (EEE), Ahsanullah University of Science \& Technology (AUST). Since joining AUST in 2004, he served in various positions at the department, faculty and university levels. He received the B.Sc. (1st class Honors) and M.Sc. (1st class Honors) in Electrical \& Electronic Engineering from Ahsanullah University of Science \& Technology and Bangladesh University of Engineering \& Technology respectively .Now he is continuing his Ph.D. degree in Electrical \& Electronic Communication Engineering at Bangladesh University of Engineering \& Technology. His research interests include Opto-electronics \& Photonics, Optical Fiber Communication Systems, Optical Networks, Soliton propagation, Satellite Communications, Mobile and Infra-red communications with over 25 publications. 